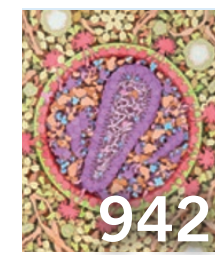

State of the art:

Innovators meld

design and

biomedicine

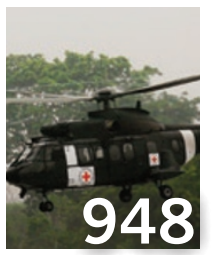

On the frontlines:

The challenges of

delivering medicines

in a war zone

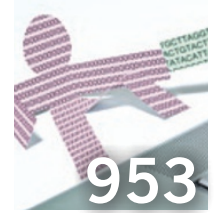

DIY genetics:

Citizen scientists

crowd-source

genetic research

\title{
Lack of uptake threatens promise of HIV vaccines
}

The news out of Thailand last year that an experimental vaccine reduced the risk of contracting HIV by as much as a third reinvigorated hopes that a vaccine against the elusive virus was possible. But new research showing that large swaths of the public wouldn't bother getting immunized against HIV, were such a vaccine available, calls into question the effectiveness of the approach in controlling the AIDS epidemic.

"Even if you've got a vaccine, it doesn't magically get into the people who need it," says Peter Newman, a social science researcher at the University of Toronto. "If we put our heads in the sand, then when an HIV vaccine comes out, we're going to hit a lot of walls unless there have been good public awareness campaigns."

Around \$1 billion is spent annually on HIV vaccine research, and there are currently around 30 vaccines in early- to mid-stage clinical trials. The latest trial was announced last month by the Dutch biotech Crucell, which plans to test a two-shot combination of vaccine candidates in healthy adults in the US and Africa. But according to Newman, who studies the acceptability of HIV biomedical prevention tools, the rewards of this effort could be limited in the absence of a more ardent public health education strategy.

Newman and his graduate student Carmen Logie conducted a meta-analysis of 20 studies involving more than 7,500 people evaluating their stated willingness to receive a hypothetical HIV vaccine. Reporting in July, the researchers found that, on average, around $65 \%$ of study participants said they would get an HIV vaccine, but only around $40 \%$ were prepared to get immunized if such a yet-tobe-developed vaccine was only moderately effective against preventing infection (AIDS 24, 1749-1756, 2010).

The study "uncovers the challenges we're going to have once we're ready to roll out an HIV vaccine into the community," says Steffanie Strathdee, associate dean of global health sciences at the University of CaliforniaSan Diego School of Medicine, who was not involved in the research. "We need to make sure communities understand that a vaccine

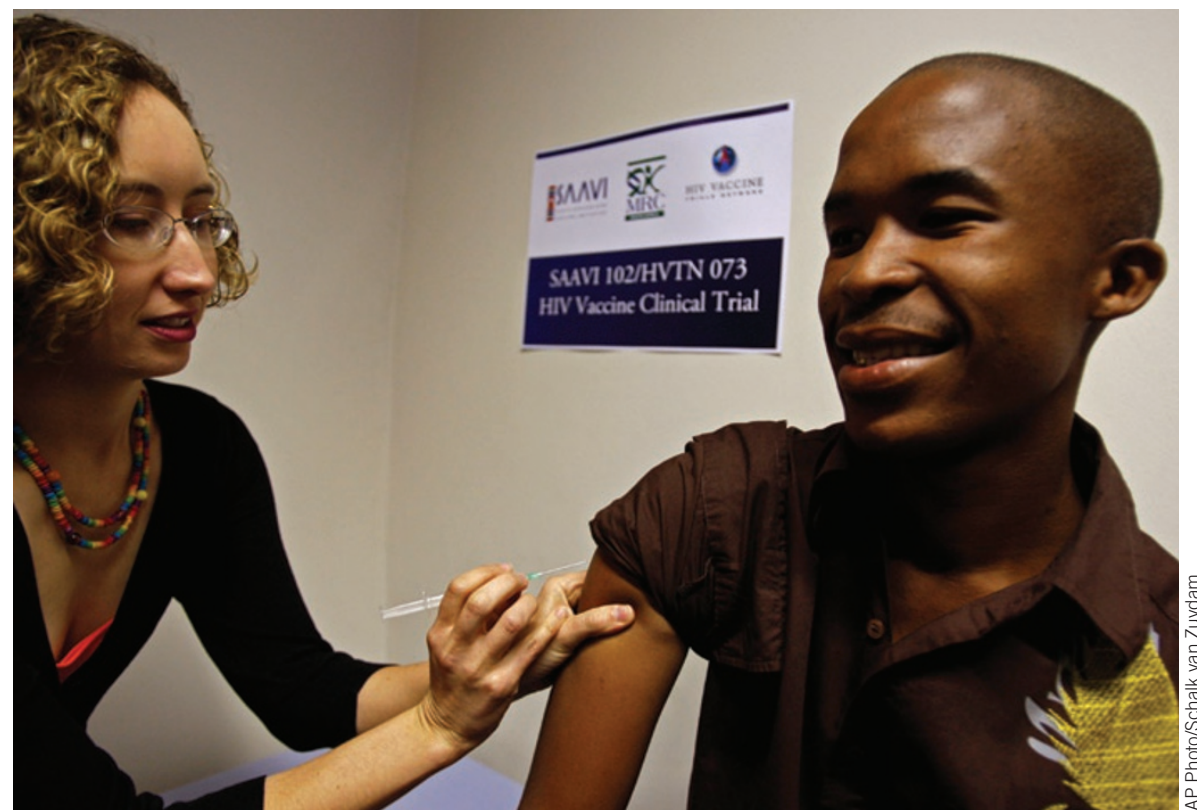

Give it a shot: Acceptance is crucial for an HIV vaccine to be effective.

is one component of a comprehensive prevention strategy," which should also include microbicides, needle exchange programs and antiretroviral therapy, among other interventions.

Even though fewer than half of the respondents said they'd take a partially effective vaccine, Seth Berkley, president and chief executive officer of the International AIDS Vaccine Initiative, actually thinks that's "a pretty good starting point."

"You never go from zero to $100 \%$ coverage with any vaccine; you always have a slow uptake," notes Berkley, who coauthored a paper in July calling the last year a "renaissance" in HIV vaccine development (N. Engl. J. Med. 363, e7, 2010). After public health officials prove that a non-theoretical vaccine is safe and effective, "then you'd start to see acceptance and that's what we've seen with every other vaccine," he says.

Given that all but three of the 20 studies included in the analysis were conducted in North America, some researchers question how widely applicable the findings are in regions of the world where HIV is more prevalent. "There's a need for more studies in high-incidence populations," says Katherine Kripke, assistant director of the vaccine research program in the US National Institute for Allergies and Infectious Diseases' division of AIDS in Bethesda, Maryland. "I think the issues might be different in places like South Africa than they are here [in the US]." (The study with the highest rate of acceptability in Newman's analysis, 94\%, was carried out in Uganda.)

Indeed, after Merck's STEP trial was halted in 2007 because the vaccine seemed to increase the risk of HIV infection, many people in Africa were still clamoring to sign up for the next vaccine trial, according to Alan Bernstein, executive director of the New York-based Global HIV Vaccine Enterprise. "The people in the communities have said, 'please, we need a vaccine."

For his part, Newman says he is now applying for funding to repeat the study's questionnaires in African countries that are hardest hit by the epidemic.

Elie Dolgin 